\title{
Моделирование линейных датчиков Холла во временной области и экспериментальная идентификация параметров их моделей с использованием оборудования компании Rohde\&Schwarz
}

\author{
Н. Лемешко, д. т. н. ${ }^{1}$, П. Струнин ${ }^{2}$
}

УДК 535.131 | ВАК 05.11.01

\begin{abstract}
Разработка электронных устройств различной функциональности обычно в той или иной степени связана с использованием математического моделирования [1]. Оно является эффективным инструментом исследования, если модели отвечают предъявляемым требованиям по точности и имеют достаточно широкие границы адекватности. При проектировании электронных устройств наиболее часто используют поведенческие и схемные модели, позволяющие оценивать их функциональность на основе виртуального прототипа, который строится путем объединения моделей электронных компонентов в соответствии с фундаментальными законами теории электрических цепей. Моделирование позволяет избежать серьезных проектных ошибок и снизить стоимость процесса проектирования. В статье рассматриваются подходы к моделированию пассивных и активных датчиков Холла (ДХ), а также экспериментальное определение значений постоянных времени моделей дХ с использованием оборудования компании Rohde\&Schwarz.
\end{abstract}

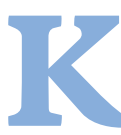
настоящему времени созданы и широко применяются модели наиболее распространенных типов электронных компонентов. Среди всей совокупности компонентов в части моделирования особого внимания заслуживают датчики неэлектрических величин [2], в частности, датчики Холла, широко применяемые для обнаружения и измерения уровня магнитных полей низкой и средней интенсивности. Предлагаемые на рынке датчики можно подразделить на имеющие цифровой выход, напряжение на котором меняется скачком при достижении заданного уровня магнитного поля, и линейные, для которых данное напряжение пропорционально напряженности магнитного поля с точностью до постоянной составляющей. В дальнейшем мы будем рассматривать линейные Дх как наиболее универсальные, а также модели для них, имея в виду, что реализация свойственных Дх с цифро-

АО «Корпорация "Комета», ведущий научный сотрудник, nlem83@mail.ru.

ООО «РОДЕ И ШВАРЦ РУС", директор департамента продаж,

Pavel.Strunin@rohde-schwarz.com. вым выходом пороговых преобразований может быть легко промоделирована с использованием подходов, изложенных в [3].

Одной из перспективных областей использования линейных ДХ является получение в реальном времени информации о текущем уровне магнитного поля, необходимой для построения активных магнитных экранов, все шире применяемых для целей обеспечения электромагнитной совместимости. Суть активного экрана состоит в том, что внешнее магнитное поле компенсируется специальной системой электромагнитов, которая формирует противоположное по направлению поле равной напряженности [4]. Таким подходом достигается устранение необходимости использования тяжелых экранов большой толщины, выполняемых из ферромагнетиков, которые по ряду причин обладают невысокой эффективностью, а также существенно ухудшают массогабаритные характеристики электронных устройств [5]. Компенсируемое магнитное поле в общем случае меняется по произвольному закону, а также по направлению, поэтому система активного экранирования включает обычно три взаимно ортогональных магнитных датчика. 
Для моделирования такой системы, а также в ряде других случаев должны использоваться динамические модели ДХ, отражающие их реальные инерционные свойства. Практика показала, что выходное напряжение Дх по отношению к синусоидальному магнитному полю неизбежно имеет амплитудно-фазовые искажения, понимание природы которых важно для построения моделей ДХ.

\section{ЭФФЕКТ ХОЛЛА И ПРИЧИНЫ ВОЗНИКНОВЕНИЯ АМПЛИТУДНО-ФАЗОВЫХ ИСКАЖЕНИЙ}

Известно, что эффект Холла заключается в возникновении поперечной разности потенциалов на боковых гранях пластины, по которой протекает электрический ток. Суть явления поясняет рис. 1а, на котором изображена упрощенная конструкция ДХ. Он представляет собой четырехполюсник, обычно выполненный в виде тонкой пленки из полупроводникового материала. Токовые электроды 1 и 2 выполняются по всей ширине поперечных граней, что обеспечивает равномерное распределение входного тока по сечению пленки. Потенциальные (холловые) электроды 3 и 4 расположены в центральной части продольных граней.

В магнитном поле носители заряда под действием сил Лоренца изменяют свою траекторию, вследствие чего на одной из боковых граней концентрация зарядов одного знака увеличивается, а на противоположной грани - уменьшается. Возникающая при этом разность потенциалов (электродвижущая сила Холла) определяется выражением [2]

$$
E_{\text {хл }}=R_{\text {хл }} \varphi\left(K_{\Gamma}, \theta\right) \operatorname{IB} \cos (\alpha) / d \text {, }
$$

где $\mathrm{R}_{\text {хл }}$ - постоянная Холла, зависящая от свойств пленки; $\varphi\left(K_{\Gamma}, \theta\right)$ - функция, зависящая от геометрии преобра- зователя и так называемого угла Холла между векторами плотности тока и напряженности суммарного электрического поля, определяемого подвижностью носителей заряда и значением магнитной индукции; $\alpha$ - угол между векторами магнитной индукции и магнитной осью ДХ, обычно совпадающей с нормалью к плоскости пленки, $d$ - толщина пленки. Формула (1) хорошо описывает формирование выходного напряжения Дх в статическом режиме, когда ток I и магнитная индукция В не изменяются во времени.

Особенно сильно эффект Холла проявляется в германии, кремнии и полупроводниках, состоящих из элементов III и V групп периодической системы. Для полупроводниковых материалов значение $\mathrm{R}_{\text {хл }}$ составляет $10^{-4}-10^{-2} \mathrm{M}^{3}$ / (A· с), в то время как для чистых металлов, например для меди, $R_{\text {хл }}=6 \cdot 10^{-11} \mathrm{M}^{3} /(\mathrm{A} \cdot \mathrm{c})$. Толщина пленок $d$ составляет обычно 0,01-0,2 мм, они вырезаются из монокристаллов и шлифовкой доводятся до требуемой толщины, а затем приклеиваются на подложку из радиотехнической слюды или ситалла [2].

Для ДХ на основе полупроводников на первое место выходит проблема повторяемости характеристик от образца к образцу, и геометрическая конфигурация пленки ДХ должна выбираться такой, чтобы технологические погрешности оказывали минимальное влияние на фактический коэффициент преобразования, который, как следует из (1), определяется произведением $\mathrm{R}_{\text {хл }} \varphi\left(\mathrm{K}_{\Gamma}, \theta\right)$. Исследования показали, что для некоторых конфигураций пленки, например, показанной на рис. 1б, значение $\varphi\left(K_{\Gamma}, \theta\right) \approx 1$ при $l / b=2$ и $a / l \leq 0,1$. Представленная на рис. 16 конфигурация пленки снижает входное и выходное сопротивление ДХ, упрощает их изготовление и уменьшает степень гальванической связи между входом и выходом датчика. Далее мы будем рассматривать ДХ, для которых

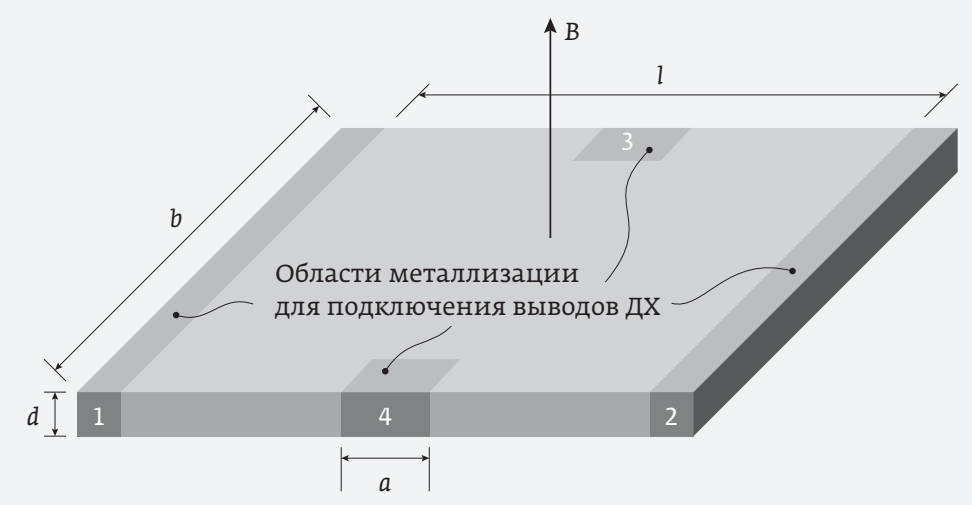

a)

б)

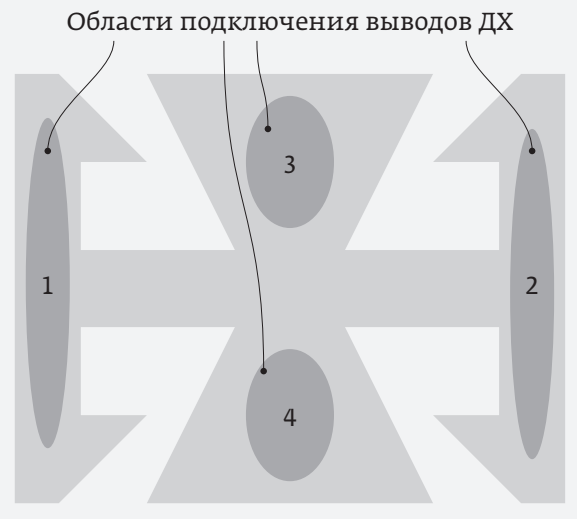

Рис. 1. Конструкция дХ: а - классическая; 6 - применяемая для производства современных датчиков 
названное условие выполняется, считая, что коэффициент преобразования определяется только значением $\mathrm{R}_{\text {хл }}$.

Динамические характеристики области пленки ДХ, в которой наблюдается эффект Холла, могут быть оценены временем релаксации $\tau=\varepsilon / \gamma$, где $\varepsilon-$ диэлектрическая проницаемость, $\gamma$ - удельная проводимость материала пленки. Для традиционно применяемых материалов $\tau=10^{-13}-10^{-11}$ с, поэтому теоретически постоянная Холла не должна зависеть от частоты вплоть до 100 ГГц. Межэлектродные емкости у ДХ составляют единицы пикофарад, поэтому их влияние проявляется на частотах порядка 100 МГц. Таким образом, формирование разности потенциалов при эффектеХолла является процессом с весьма малой инерционностью. Оцененные в [6] собственная емкость рабочей области ДХ из полупроводникового материала и его граничная частота составляют 0,7 пФ и 100 МГц соответственно, но и такая частота не может быть принята в качестве характеристики быстродействия ДХ, Т. К. здесь имеются ограничения иного рода.

В [2] отмечается, что при работе ДХ в переменных магнитных полях в выходной цепи ДХ появляется дополнительная электродвижущая сила (ЭДС), индуцируемая непосредственно магнитным полем. Для синусоидального поля с частотой $f$ ее максимум составит $E_{\text {инд, }}=2 \pi f \mathrm{~B}_{\mathrm{m}} S$, где $B_{m}$ - максимум индукции, $S$ - площадь контура, пронизываемого магнитным потоком, и она будет сдвинута по отношению к ЭДС Холла на 90. Собственно, этот эффект и является источником амплитудно-фазовых искажений сигнала на выходе ДХ по отношению к внешнему синусоидальному полю. Уменьшение индуцируемых ЭДС осуществляется рациональным расположением выводов ДХ, а также использованием, при необходимости, компенсационных обмоток.

В переменном магнитном поле в пленке дх возникают вихревые токи, магнитное поле которых изменяет основное поле и тем самым ЭДс Холла. Вектор наведенного магнитного поля сдвинут относительно вектора индукции внешнего поля примерно на 90, и поэтому изменение ЭДС Холла происходит не только по значению, но и по фазе. Вихревые токи приводят также к разогреву пленки ДХ.

В [2] приводится расчетное соотношение, аккумулирующее названные физические эффекты. При питании ДХ постоянным током и нахождении его в переменном синусоидальном поле с индукцией до 0,5 Тл зависимость ЭДС Холла от частоты и времени имеет вид

$$
\dot{\mathrm{E}}_{\text {Хл }}(t)=\mathrm{R}_{\text {Хл }} \mathrm{IB} \sqrt{1+\frac{\left(2 \pi f \mu \gamma b^{2}\right)^{2}}{48}} e^{j(2 \pi f t+\varphi)} / d,
$$

где $\mu$ - абсолютная магнитная проницаемость среды, окружающей преобразователь, В - амплитуда индукции; $\varphi$ - фазовый сдвиг, который также зависит от частоты:

$$
\varphi=\operatorname{arctg}\left(2 \pi f \mu \gamma b^{2} / c\right)
$$

где коэффициент $c=8$. Представленные соотношения получены на основе физической модели ДХ и проверены путем соответствующих измерений. Они достаточны для построения динамической модели ДХ в виде математических соотношений, однако для ее формализации в виде простой схемной модели, применимой в системах схемного моделирования, целесообразно согласовать частотные зависимости амплитуды и фазы ЭдС Холла путем задания значения с равным $\sqrt{48} \approx 6,93$. Расчеты показали, что такая замена вносит фазовую погрешность не более 4,1, или эквивалентную ей максимальную амплитудную погрешность не выше 6,8\%. В классическом моделировании погрешность до $\pm 10 \%$ считается приемлемой.

\section{СХЕМНЫЕ МОДЕЛИ ДАТЧИКОВ ХОЛЛА}

Как следует из формул (1) и (2), эффект Холла при воздействии магнитного поля средней интенсивности создает напряжение на уровне 0,1-10 мВ, которое трудно использовать в практических целях. По этой причине многие линейные Дх дополнены усилительными блоками, которые имеют свои фазовые и амплитудные характеристики. Эти классы ДХ отличаются по назначению, и их модели будут разными по структуре. Построение моделей осуществлялось на основе предположения, что вектор магнитной индукции перпендикулярен к плоскости чувствительного элемента ДХ.

Для пассивных ДХ может быть использована модель, представленная в схемном виде на рис. 2. Пары резисторов $\mathrm{R}_{1}$ и $\mathrm{R}_{2} ; \mathrm{R}_{3}$ и $\mathrm{R}_{4}$ моделируют входное и выходное сопротивления ДХ $\mathrm{R}_{\mathrm{BX}}$ и $\mathrm{R}_{\text {вых }}$ соответственно, причем с учетом симметрии (см. рис. 1б) $R_{1}=R_{2}=0,5 R_{\text {BX, }} R_{3}=R_{4}=0,5 R_{\text {вых }}$. Емкостью $C_{D}$, которая моделирует инерционные свойства рабочей области ДХ, в модели следует пренебречь, т.. на частотах, на которых ее влияние существенно, ДХ не используются. Зависимость индукции магнитного поля от времени $\mathrm{B}(t)$ вводится в модель как напряжение $u_{2}(t)$ источника $E_{1}$, что соответствует развитой в [7] теории функционально-интерфейсных моделей, согласно которой управляющие воздействия и неэлектрические физические величины могут вводиться в схемные модели как эквивалентные токи или напряжения, если моделирование осуществляется во временной области.

Цепь $\mathrm{R}_{f}-\mathrm{L}_{f}$ предназначена для моделирования рассмотренных выше амплитудно-фазовых искажений, возникающих при воздействии переменного магнитного поля на ДХ. Комплексное сопротивление такой цепи имеет вид

$$
\begin{aligned}
\dot{Z}=R_{f} & +j 2 \pi L_{f} f=\sqrt{R_{f}^{2}+\left(2 \pi L_{f} f\right)^{2}} e^{j a r c t g\left(2 \pi f L_{f} / R_{f}\right)}= \\
& =R_{f} \sqrt{1+\left(2 \pi L_{f} f / R_{f}\right)^{2}} e^{j a r c t g\left(2 \pi f L_{f} / R_{f}\right)} .
\end{aligned}
$$


Передаточная функция управляемого источника тока Ј имеет вид $I_{J}(t)=k u_{1}(t) u_{2}(t)$, где $u_{2}(t)=B(t), k-$ некоторый коэффициент, причем $u_{1}(t)=I_{B X}(t) R_{B X}$ в случае, если вход и выход ДХ не имеют внешней гальванической связи. При наличии последней входной ток может претерпевать изменения, однако модель адекватности не теряет, т.к. соответствует конструкции ДХ. Для удобства сопоставления с формулой (2) будем считать входной ток постоянным и равным I. Напряжение $u_{3}(t)$

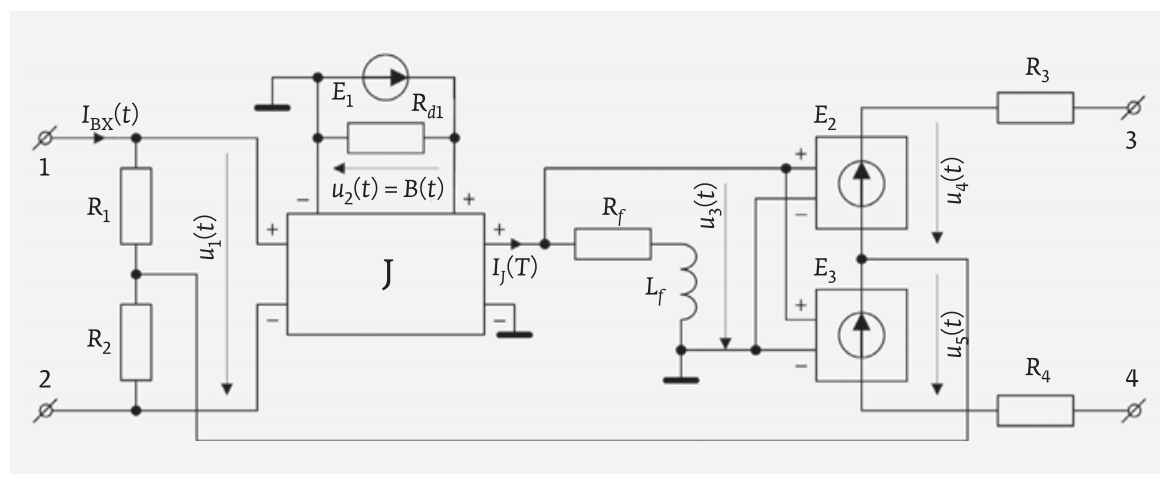
в модели соответствует ЭДС Холла и в комплексном виде при нулевой фазе синусоидального магнитного поля с амплитудной индукцией В рассчитывается следующим образом:

$$
\dot{u}_{3}(t)=k R_{\mathrm{BX}} R_{f} I B \sqrt{1+\left(2 \pi L_{f} f / R_{f}\right)^{2}} e^{j\left(2 \pi f t \operatorname{arctg}\left(2 \pi f L_{f} / R_{f}\right)\right)} .
$$

Сопоставляя уравнения (2), (3) и (5), можно прийти к выводу, что в рассмотренной модели должны выполняться условия $k \mathrm{R}_{\mathrm{BX}} \mathrm{R}_{\mathrm{f}}=\mathrm{R}_{\mathrm{xл} ;} \mathrm{L}_{\mathrm{f}} / \mathrm{R}_{\mathrm{f}}=\mu \gamma b^{2} / \sqrt{48}$. Для дальнейшего использования введем параметр $\tau=L_{f} / R_{f}$ как единую характеристику амплитудно-фазовых искажений трансформации индукции магнитного поля в ЭДС Холла, возникающих только из-за магнитных эффектов. Справедливость уравнения (5) для гармонической зависимости $\mathrm{B}(t)$ и отсутствие каких-либо иных ограничений позволяет говорить об универсальности построенной модели, в Т. ч. при питании ДХ переменным током. Напряжения $u_{4}(t)$ и $u_{5}(t)$, формируемые управляемыми источниками $E_{2}$ и $E_{3}$, равны половине напряжения $u_{3}(t)$. Сопротивление $\mathrm{R}_{d l}$ необходимо для замкнутости контура протекания тока источника $\mathrm{E}_{1}$ и может иметь любой номинал.

Отличия активных ДХ от пассивных заключаются в следующем:

- они не имеют внешнего токового входа, а сам датчик питается постоянным током;

- в них имеется встроенный дифференциальный усилитель, обычно имеющий не менее двух каскадов, в Т. ч. выходной сравнительно высокой мощности;

- выходное напряжение активного ДХ строго пропорционально индукции постоянного магнитного поля в установленных пределах линейности;

- в отсутствие внешнего магнитного поля выходное напряжение обычно равно половине напряжения электропитания;

- искажению за счет нежелательных магнитных эффектов в наибольшей степени подвергается напряжение на входе усилителя как имеющее малый

уровень, а в каскадах дифференциального усилителя такими искажениями допустимо пренебречь.

Эти качественные предпосылки и следует положить в основу построения модели активного ДХ, учитывая, что амплитудно-фазовым искажениям будет подвергаться непосредственно напряжение на выходе пассивной части Дх до усилителя. В качестве характеристики преобразования постоянного магнитного поля в выходное напряжение для таких дх будем использовать чувствительность $S$, имеющую единицу измерения В / Тл или В / Гс. Согласно определению, значение $S$ характеризует приращение напряжения $\mathrm{U}_{\text {вых }}$ на выходе ДХ при изменении силовой характеристики постоянного магнитного поля на единичное значение, т. е., например, $S=d U_{\text {вых }} / d B$. В дальнейшем будем считать, что значение $S$ измеряется в В / Гс (1 Тл = 10000 Гс), поскольку эта единица применяется в справочниках по магниточувствительным измерительным преобразователям [2]. Например, для используемого ниже в качестве объекта исследований датчика SS495A-SP она имеет значение 3,125 мВ / Гс

Исходя из этого, для активных ДХ может быть предложена следующая модель (рис. 3). Магнитная индукция как функция времени задается источником $\mathrm{E}_{S 1}$. Ток источника J равен $\mathrm{I}_{\mathrm{J}}(t)=k u_{1}(t)$. Комплексное напряжение $u_{2}(t)$, учитывающее вносимые пассивной частью ДХ амплитудно-фазовые искажения, при синусоидальном характере магнитного поля будет иметь вид

$$
\dot{u}_{2}(t)=k R_{f} B \sqrt{1+(2 \pi f \tau)^{2}} e^{j(2 \pi f t+a r c t g(2 \pi f \tau))},
$$

откуда следует требование выполнения условия $\mathrm{kR}_{\mathrm{f}}=\mathrm{S}$.

Блоки $A_{1}, \ldots, A_{n}$ моделируют амплитудно-частотные свойства дифференциального усилителя в составе активного Дх. Каждый из них имеет единичный коэффициент передачи на постоянном токе и характеризуется постоянной времени $\tau_{i}=\mathrm{R}_{i} \mathrm{C}_{i}$. Можно показать, что комплексное напряжение $u_{k n}(t)$ на выходе $n$ каскадов будет определяться выражением 


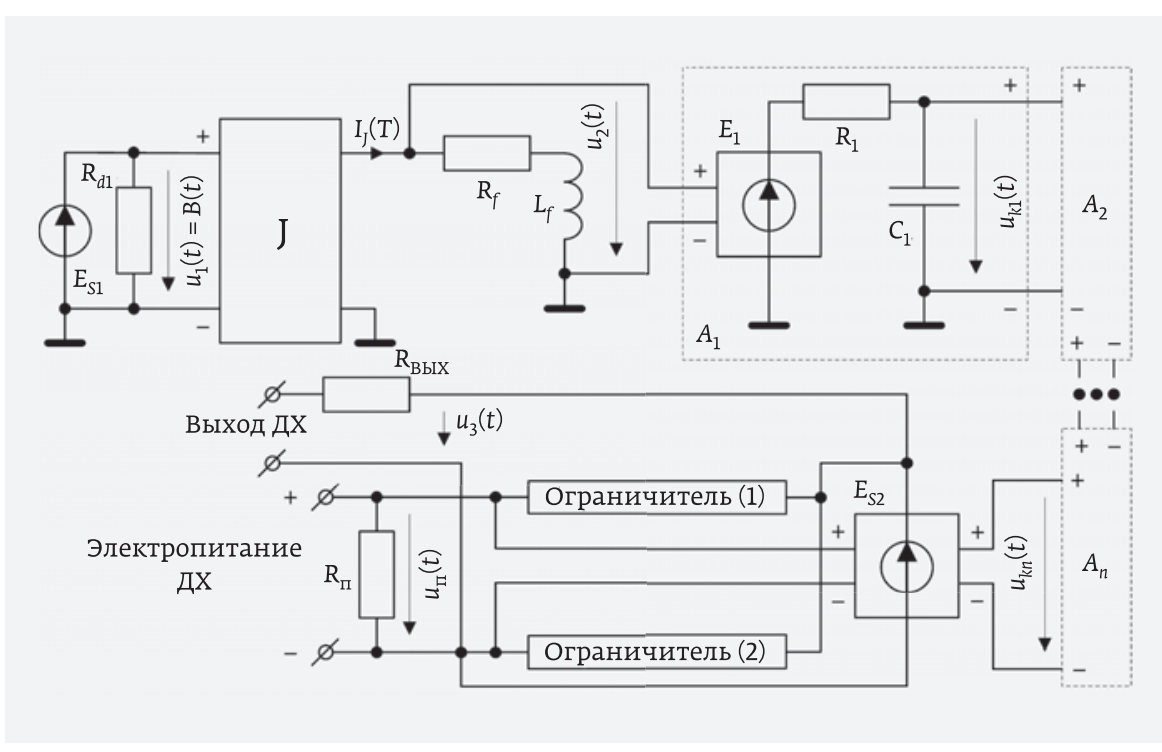

Рис. 3. Модель активного дХ

$$
\dot{u}_{k n}(t)=\dot{u}_{2}(t) \exp \left(-j \sum_{i=1}^{n} \operatorname{arctg}\left(2 \pi f \tau_{i}\right)\right) \cdot \prod_{i=1}^{n} \frac{1}{\sqrt{1+\left(2 \pi f \tau_{i}\right)^{2}}} .
$$

Передаточные функции по амплитуде и фазе для модели активного ДХ имеют вид:

$$
\begin{gathered}
A(f)=S B_{m} \sqrt{1+(2 \pi f \tau)^{2}} \prod_{i=1}^{n} \frac{1}{\sqrt{1+\left(2 \pi f \tau_{i}\right)^{2}}} ; \\
\Phi(f)=\operatorname{arctg}(2 \pi f \tau)-\sum_{i=1}^{n} \operatorname{arctg}\left(2 \pi f \tau_{i}\right) .
\end{gathered}
$$

Уравнения (8) являются основой для идентификации параметров, отражающих динамические свойства модели активного ДХ. Напряжение $u_{3}(t)$ на выходе источника $E_{s 2}$ в линейном режиме составляет $u_{3}(t)=u_{k n}(t)+0,5 u_{\pi^{\prime}}$ если в отсутствие внешнего магнитного поля выходное напряжение ДХ равно половине напряжения электропитания. Сопротивление $\mathrm{R}_{\text {п }}$ служит для моделирования потребления тока схемой ДХ.

Предложенная модель адекватно отражает свойства активных ДХ в таком диапазоне индукции, в котором сохраняется постоянство значения $S$. Для моделирования режима отсечки, свойственного активным ДХ при воздействии чрезмерно интенсивного магнитного поля, в схему включены два ограничителя, причем ясно, что напряжение на выходе не может быть более $u_{п}$ и менее 0 В. В общем случае пределы ограничения определяются конкретным типом датчика Холла и, например, для SS495A-SP составляют 0,5 и 4,5 В при напряжении питания 5 В. Примеры моделей ограничителей напряжения приведены в [3]. Для некоторых типов активных Дх может потребоваться замена емкости в модели усилительного каскада на RC-цепь для моделирования работы в области высоких частот, например, если значение коэффициента усиления составляет порядка единицы. Это приведет кпоявлению еще одной постоянной времени для каждого каскада в модели.

Для рассмотренных моделей температурная нестабильность ДХ учитывается изменением коэффициентов $\mathrm{R}_{\text {хл }}$ либо $\mathrm{S}$. Модели являются универсальными и позволяют моделировать работу Дх для любой функции $\mathrm{B}(\mathrm{t})$

\section{ПРИНЦИПЫ ИДЕНТИФИКАЦИИ ПАРАМЕТРОВ МОДЕЛЕЙ ДАТЧИКОВ ХОЛЛА}

Для обеспечения соответствия свойств модели конкретному образцу необходимо идентифицировать ее параметры. Известно [1], что это может быть сделано двумя способами расчетным и экспериментальным. В случае Дх такие параметры, как входное и выходное сопротивление, а также постоянная Холла измеряются тривиальным образом, а в некоторыхслучаях приводятся как справочные данные. Наибольший интерес представляет вопрос об идентификации динамическиххарактеристик активных ДХ, информация о которых, как правило, отсутствует.

Поскольку количество усилительных каскадов в активном ДХ не известно и не может быть определено внешними измерениями, то целесообразно выбирать его из следующих соображений. Выходной каскад всегда имеет отличия от предыдущих, и поэтому в составе модели дифференциального усилителя должно быть не менее двух блоков (см. рис. 3), т. е. целесообразно установить, что n=2. Тогда в рамках идентификации динамических характеристик модели необходимо найти значения $\tau, \tau_{1}$, $\tau_{2}$ на основе измерений, выполненных для конкретного образца активного Дх при его работе в линейном режиме. Как следует из уравнений (8), в ходе таких измерений должны быть определены значения амплитуды сигнала на выходе датчика и фазовых сдвигов между гармоническим магнитным полем и выходным напряжением Дх не менее чем для трех частот. Базовые положения в части получения экспериментальных данных и расчета постоянных времени состоят в следующем.

1. Датчик Холла должен размещаться в однородном по структуре и напряженности магнитном поле. Известно, что внутри объема соленоида, наиболее подходящего для создания тестового магнитного поля, это условие будет соблюдаться примерно на трети длины его центральной части, если диаметр соленоида не менее чем в три раза меньше его длины. Следовательно, такая катушка будет иметь 
достаточно большие размеры, высокую собственную емкость и индуктивность и, как следствие, сравнительно низкую резонансную частоту. Измерения следует проводить в дорезонансной области частот.

2. На частотах до 1 кГц целесообразно ограничивать ток в катушке для снижения тепловыделения, что позволит не допустить существенного изменения активного сопротивления катушки за один цикл измерений. С учетом повышения полного сопротивления соленоида с увеличением частоты генератор синусоидального сигнала должен иметь низкое выходное сопротивление.

3. Применяемое измерительное оборудование должно иметь качественно реализованную функцию измерения разности фаз, поскольку ожидаемый фазовый сдвиг между магнитным полем и выходным напряжением ДХ на частотах порядка 1 кГц составляет порядка $5^{\circ}$.

4. Предварительные расчеты показали, что система уравнений, построенная на основе формул (8), в подавляющем большинстве случаев не будет иметь точного решения. Значения $\tau, \tau_{1}, \tau_{2}$ следует определять с использованием функций поиска приближенного решения, например, реализованных в программе Mathcad 15

Далее рассмотрим результаты идентификации динамических параметров ДХ, выполненной с учетом этих предпосылок.

\section{ПРИМЕР ВЫПОЛНЕНИЯ ИЗМЕРЕНИЙ}

\section{ДЛЯ ИДЕНТИФИКАЦИИ ДИНАМИЧЕСКИХ} ПАРАМЕТРОВ ДХ С ИСПОЛЬЗОВАНИЕМ ОБОРУДОВАНИЯ КОМПАНИИ RОНDE\&SCHWARZ Объект исследований, оборудование и измерительная оснастка. Для проведения измерений был выбран линейный активный ДX SS495A-SP фирмы Honeywell, предназначенный для измерений индукции магнитного поля в диапазоне значений до 600 Гс [8]. При напряжении электропитания, равном 5 В, в отсутствие внешнего магнитного поля его выходное напряжение составляет $(2,5 \pm 0,075)$ В, погрешность чувствительности - не более 0,125 мВ/Гс, температурный дрейф не выше 0,06\%/ ${ }^{\circ} \mathrm{C}$. Функциональная схема ДХ, в общем случае не известная и не требующаяся для идентификации динамических параметров, для ДX SS495A-SP изображена на рис. 4а, передаточная характеристика - на рис. 46 [8].

Использованный принцип измерений состоял в том, что образец ДХ помещался в катушку индуктивности без сердечника, удовлетворяющую требованиям по однородности магнитного поля. Питание катушки осуществлялось переменным синусоидальным током перестраиваемой частоты от источника с малым выходным сопротивлением. Для разных значений частоты снимались амплитудные значения тока в катушке и напряжения на выходе генератора, а также разности фаз между ними. Эти данные являются основой для дальнейшей идентификации параметров ДХ.

При выполнении эксперимента использовалась схема, представленная на рис. 5. Для автоматических измерений амплитудно-фазовых характеристик сигналов использовался осциллограф R\&S RTO1044 с полосой 4 ГГц и четырьмя каналами. На канал 1 осциллографа подавалось напряжение с балластного сопротивления $\mathrm{R}_{6}$ номиналом 4,9 Ом (погрешность не более 0,1 Ом), включенного последовательно с катушкой и необходимого для получения информации о форме тока в ней. Сопротивление было изготовлено в виде пары параллельно соединенных резисторов С5-37-5 номиналом 10 Ом. На канал 2 осциллографа подавался сигнал с выхода Дх. Питание цепи в составе балластного сопротивления и катушки осуществлялось от усилителя мощности в составе генератора АНР-1041 с выходным сопротивлением около 2,5 Ом. На его вход подавалось выходное напряжение генератора R\&S HMF2550, предназначенного для формирования сигналов стандартных и произвольных форм.

При проведении измерений ДХ, выполненный в виде микросхемы размерами 3 ×4 мм, размещался при помощи специального держателя в геометрическом центре катушки. Электропитание ДХ напряжением 5 В осуществлялось

a)
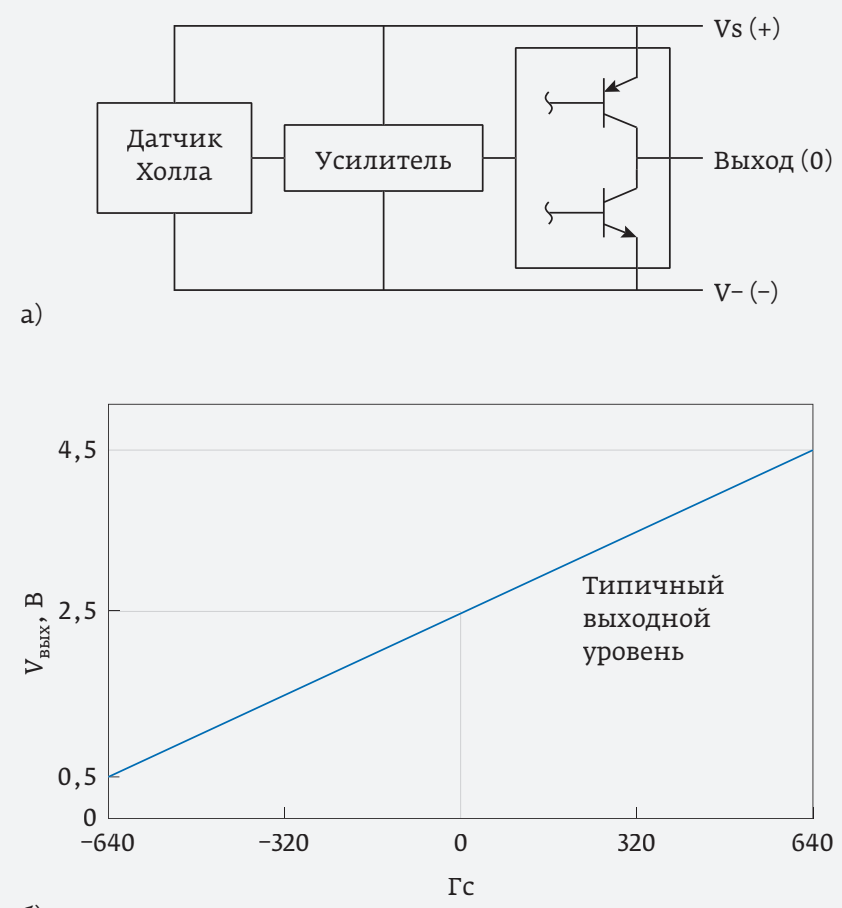

б)

Рис. 4. Активный датчик Холла SS495A-SP: a - функциональная схема; 6 - передаточная характеристика 


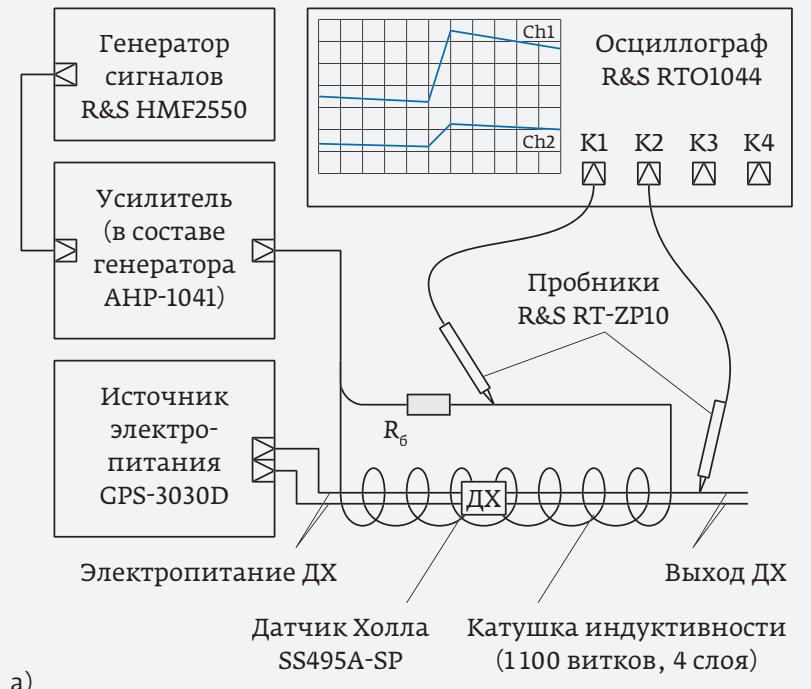

a)

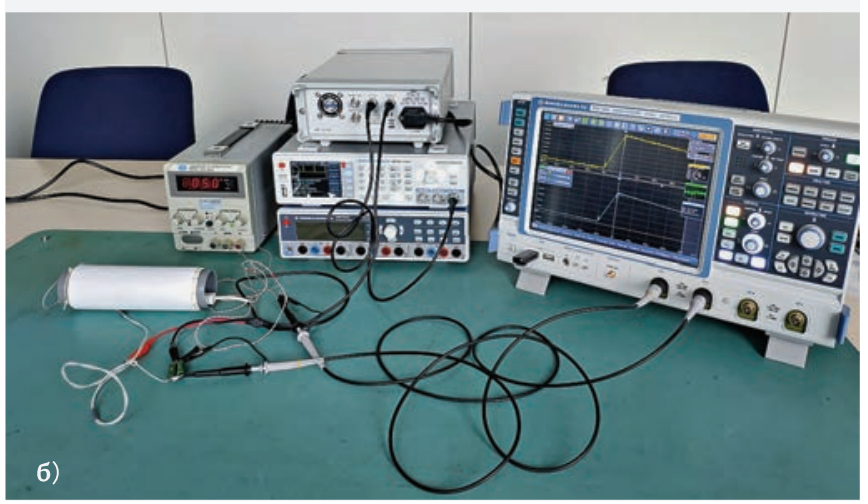

Рис. 5. Измерительная установка: а - схема; б - фотография

от лабораторного источника GPS-3030D, на фото на рис. 56 показан альтернативный вариант - программируемый источник питания постоянного тока R\&S HMP2030, характеризующийся низким уровнем пульсаций напряжения, что важно и для получения качественных результатов измерений, и для нормальной работы любых активных Дх.

Катушка индуктивности была изготовлена специально для проведения измерений. Она выполнена на пластиковом каркасе четырехслойной намоткой из провода диаметром 0,5 мм, каждый слой имеет около 280 витков при средних диаметре 53 мм и длине 146 мм. При таких характеристиках даже в отсутствие сердечника из ферромагнитных материалов расчетное значение индуктивности катушки составляет 23 мГн. Измеренное значение активного сопротивления равняется 18,5 Ом, предельно допустимый ток по критерию перегрева не выше $10{ }^{\circ} \mathrm{C}-$ не более 0,6 А. Выбранные характеристики конструкции катушки определялись необходимостью сопряжения с имеющейся приборной базой.
Подготовительные операции. Перед проведением измерений были осуществлены калибровочные процедуры, необходимые для определения нормирующего
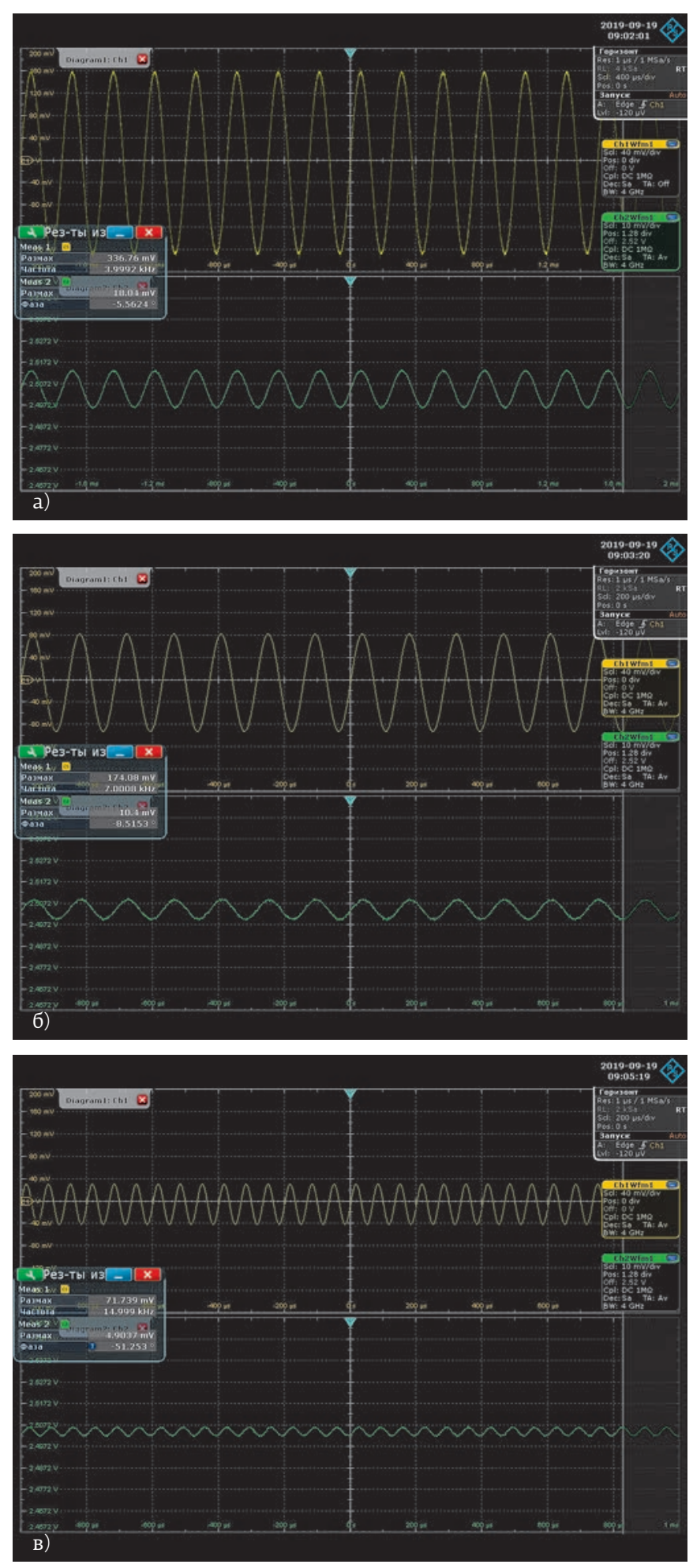

Рис. 6. Осциллограммы сигналов на балластном сопротивлении, на выходе ДХ и результаты автоматических измерений на частотах 4 кГц (а), 7 кГц (б) и 15 кГц (в) 
коэффициента трансформации тока в катушке в напряжение на выходе датчика Холла. Поскольку ДХ SS495A-SP является измерительным, с его использованием было установлено, что коэффициент катушки $k$ составляет 7130 (А/м)/A (89,6 Гс/A) при расчетном значении 7139 (А/м)/А. Оценочное значение собственной резонансной частоты катушки составляет порядка 50 кГц

Результаты измерений при формировании катушкой синусоидального магнитного поля были получены с использованием функции автоматических измерений осциллографа R\&S RTO1044 на частотах в пределах от 0,1 до 200 кГц с переменным шагом. На обоих каналах осциллографа измерялся размах напряжений и фазовый сдвиг напряжения в канале 2 относительно канала 1. Примеры осциллограмм, полученных для частот 4, 7 и 15 кГц приведены на рис. 6.

Для идентификации динамических параметров ДХ результаты измерений удобно представить сразу в нормированном виде. Для произвольной частоты обозначим размах напряжения на балластном сопротивлении $\mathrm{U}_{\mathrm{R} \sigma}$ [B] а на выходе Дх - $U_{\text {дх }}[\mathrm{MB}]$. Размах тока в катушке равен $\mathrm{U}_{\mathrm{R} \sigma} / \mathrm{R}_{6}$, соответственно, удвоенная амплитуда магнитного поля будет равна $k U_{\mathrm{R} \sigma} / \mathrm{R}_{6}$, и тогда $\mathrm{U}_{\text {дх }}=\mathrm{kSU}_{\mathrm{R} \sigma} / \mathrm{R}_{6}=57,14 \mathrm{U}_{\mathrm{R} \sigma}$ в используемых единицах измерения. Это соотношение действовало бы в случае, если внутренние цепи ДХ не проявляли бы собственные частотные свойства, которые в модели определяются постоянными времени $\tau, \tau_{1}, \tau_{2}$ С учетом изложенного, а также структуры функции $A(f)$, полученные на основе измерений значений $U_{R 6}$ и $U_{\text {дх }}$ peзультаты целесообразно представить в виде зависимости от частоты нормированной передаточной функции $A_{n}(f)=U_{\text {дх }} /\left(57,14 U_{R \sigma}\right)$. В точечном виде она представлена на рис. 7а. Как следует из приведенного графика, на частотах выше 10 кГц в катушке наблюдается резонанс токов, в результате которого функция $A_{n}(f)$ приобретает характерный выброс. Результаты измерений, полученные в резонансной области частот, в принципе могут быть скорректированы при наличии точной априорной информации о зависимости импеданса катушки от частоты, которая может быть получена, например, с использованием векторного анализатора цепей или измерителей амплитудно-частотных характеристик. В данном случае интерес представляет область частот до 10 кГц, в которой проявляется влияние побочных магнитных эффектов в датчике Холла, а резонанс в катушке еще отсутствует. На рис. 76 зависимость $A_{n}(f)$ приведена в увеличенном масштабе, на частотах в районе 4-5 кГц наблюдается характерный выброс.

На рис. 7в приведена полученная экспериментально точечная зависимость фазового сдвига $\Phi(f)$ между сигналами в каналах 2 и 1 осциллографа в диапазоне частот до 10 кГц. На основе этих данных могут быть определены значения $\tau, \tau_{1}, \tau_{2}$.

\section{РЕЗУЛЬТАТЫ ИДЕНТИФИКАЦИИ ПАРАМЕТРОВ МОДЕЛИ АКТИВНОГО ДХ}

Экспериментальные исследования были проведены для двадцати частот, из которых шесть попадают в резонансную область катушки индуктивности, и полученные для них данные не подлежат использованию для расчета значений $\tau, \tau_{1}, \tau_{2}$. В общем случае получение их оценок должно сопровождаться минимизацией ошибки аппроксимации амплитудно-частотной и фазо-частотной характеристик ДХ, и выполнению этого условия способствует

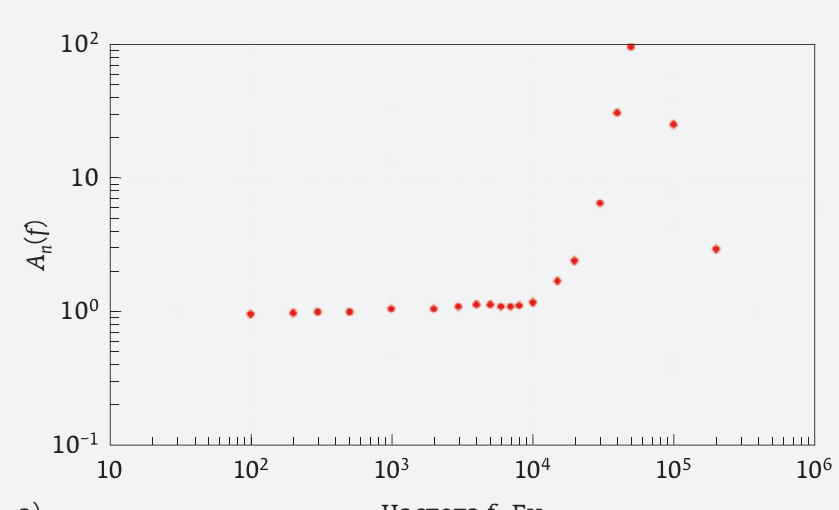

a)
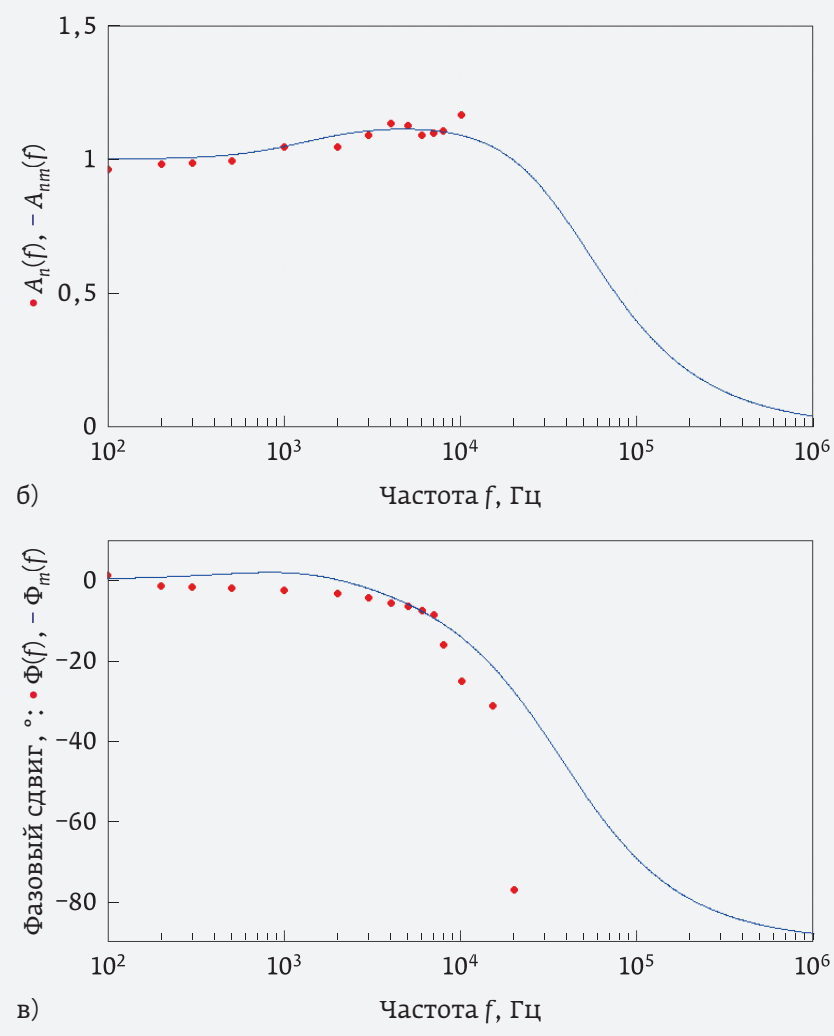

Рис. 7. Результаты измерений, полученные для активного ДХ SS495A-SP: а - зависимость $A_{n}(f)$ с резонансным выбросом; 6 - зависимость $A_{n}(f)$ в увеличенном масштабе; в - зависимость $\Phi(f)$ 
использование частотных точек, приближенно равномерно распределенных в полосе частот, где резонанс отсутствует. При этом как минимум одна из них должна соответствовать области характерного выброса.

Согласно результатам идентификации, выполненной по данным измерений для частот 0,2; 4 и 7 кГц, значения $\tau$, $\tau_{1}, \tau_{2}$ для использования в модели исследуемого ДХ составляют 0,133 мс; 4,28 мкс; 0,118 мс соответственно. На рис. 76 на построенный по точкам график функции $A_{n}(f)$ наложена кривая $A_{n m}(f)=A(f) /\left(S_{m}\right)$, построенная в соответствии с этими значениями постоянных времени. Аналогично, на рис. 7в зависимость $\Phi(f)$ дополнена рассчитанной по формуле (8) кривой $\Phi_{m}(f)$, характеризующей сдвиг по фазе между синусоидальным магнитным полем и выходным напряжением ДХ. Погрешности по фазе в интервале частот до 10 кГц не превосходят 10, по амплитуде - 7\%.

Из сопоставления расчетных и экспериментальных данных следует вывод о том, что модель активного Дх, подвергнутого измерениям, будет адекватно описывать его динамические свойства в интервале до 10 кГц. Несмотря на то, что для более высоких частот истинный вид функции $A_{n}(f)$ маскирован резонансным явлением, построенная модель будет адекватна до существенно более высоких частот, т. к. выбранные для идентификации динамических характеристик модели точки лежат строго в дорезонасной области, а причины резонанса лежат вне активного ДХ.

\section{ЭКСПЕРИМЕНТАЛЬНАЯ ПРОВЕРКА ФУНКЦИОНИРОВАНИЯ МОДЕЛИ АКТИВНОГО ДХ ПРИ ВОЗДЕЙСТВИИ ИМПУЛЬСНОГО МАГНИТНОГО ПОЛЯ}

Поскольку активные ДХ могут использоваться в качестве датчиков магнитного поля с произвольным законом изменения во времени, целесообразно проверить модель экспериментальным путем в условиях воздействия импульсного магнитного поля и при помощи такой проверки оценить необходимость использования в модели ДХ цепей, описывающих динамические свойства.

Для такой проверки использовалась та же схема измерений (см. рис. 5), но генератор сигналов R\&S HMF2550 формировал импульсы с частотой 500 Гц и длительностью 50 мкс. Для такого сигнала спектр можно считать ограниченным частотой 20 кГц. Амплитуда выходного напряжения на выходе усилителя составляла 19,2 В (на нагрузке с большим сопротивлением).

Результаты измерений для рассматриваемого случая показаны на рис. 8. Осциллограмма в верхнем поле соответствует изменению напряжения на балластном сопротивлении, в нижнем поле - напряжению на выходе датчика Холла. Осциллограмма напряжения на выходе Дх была получена при максимально доступном разрешении по вертикальному отклонению; на ней видны резонансные выбросы с частотой около 250 кГц, определяемой свойствами элементов измерительной цепи, в первую очередь катушкой индуктивности.

На рис. 9а показана расчетная форма напряжения на балластном сопротивлении $U_{R \sigma}(t)$, полученная с использованием классического гармонического синтеза суммированием 40 гармоник, амплитуда которых была рассчитана в соответствии со структурой схемы измерений. Резонансные свойства цепи питания катушки не моделировались. Для удобства сопоставления шаг и абсолютные значения координатных сеток на рис. 8 и 9 выбраны одинаковыми.

На рис. $9 б$ представлены графики, позволяющие сделать вывод о влиянии динамических свойств модели на точность моделирования работы активного ДХ. Сплошной утолщенной кривой здесь показана зависимость напряжения от времени $\mathrm{U}_{\partial}(t)$, полученная в результате моделирования, крупным пунктиром - кривая $\mathrm{U}_{\partial 1}(t)=k \mathrm{Si}(t)$, соответствующая случаю, когда динамические свойства модели неучитываются вообще. Мелким пунктиром показана кривая $U_{\partial_{2}}(t)$, построенная с учетом только постоянных времени $\tau_{1} и \tau_{2}$ и соответствующая случаю появления недопустимой погрешности в модели.

Наконец, тонкой сплошной линией с привязкой ко второй оси ординат показана функция $E(t)=\left(\left(U_{\partial}(t)-U_{\partial 1}(t)\right) /\left(U_{\partial}(t)-U_{0}\right)\right) \cdot 100 \%$, где $U_{0}=2,5$ B - постоянное напряжение на выходе дх в отсутствие внешнего электромагнитного поля. Эта функция характеризует погрешность расчета напряжения на выходе Дх при отказе от учета динамических параметров. Выполненный расчет показал, что погрешность может превышать 30\%, что существенно больше принятой для практики нормы в 10\%. Таким образом, учет динамических

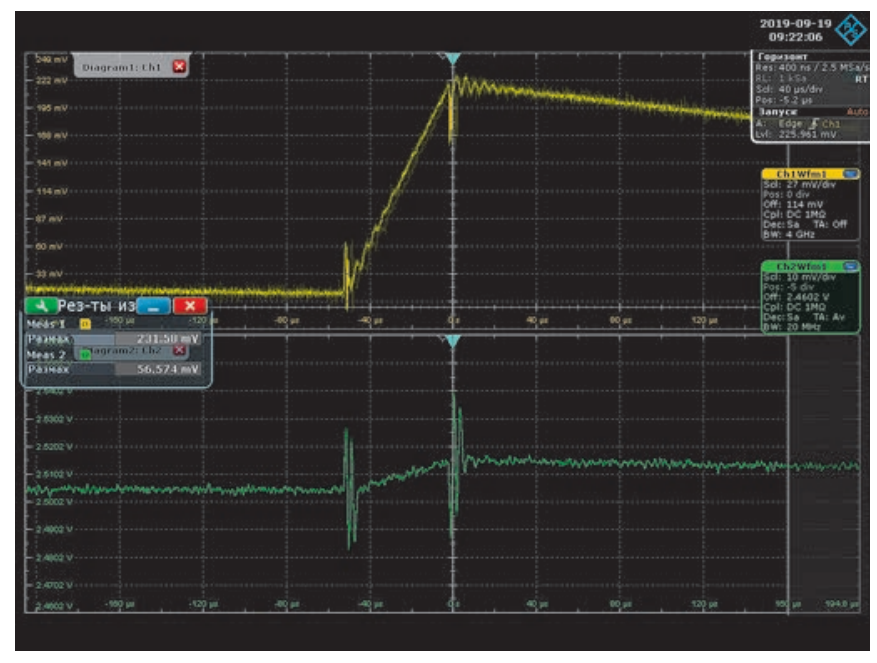

Рис. 8. Результаты, полученные на экспериментальной установке при импульсном изменении магнитного поля 

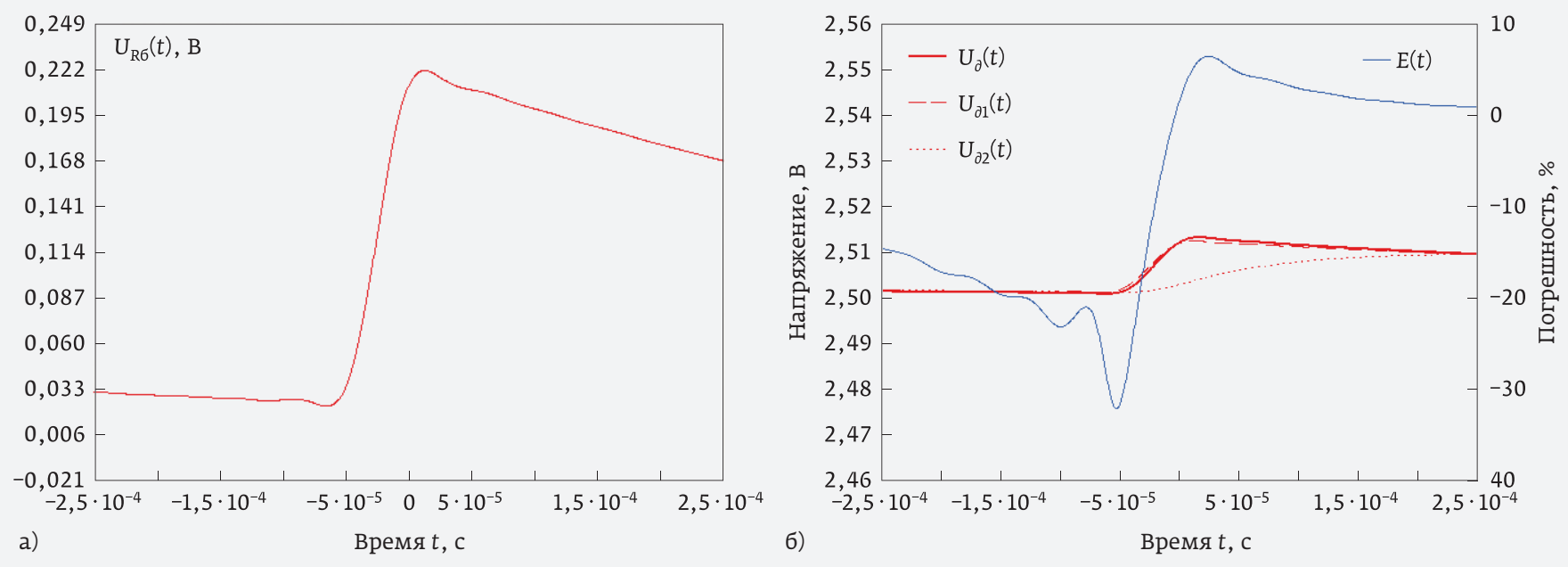

Рис. 9. Расчетные зависимости напряжений: a - на балластном сопротивлении $\mathrm{R}_{6}$; 6 - на выходе активного дХ при использовании предложенной выше модели с экспериментально идентифицированными динамическими параметрами

свойств пассивной и активной частей дх является обязательным для точного моделирования их работы в резко изменяющихся магнитных полях.

Сопоставление графиков напряжений $U_{R \sigma}(t)$ и $U_{\partial}(t)$, приведенных на рис. 8 и 9, свидетельствует о высокой точности результатов моделирования активного ДХ при использовании значений $\tau, \tau_{1}, \tau_{2}$, определенных предложенным выше способом.

$$
\therefore *
$$

Таким образом, модели активных Дх, разработанные на основе представления о физике их функционирования, получили подтверждение адекватности и точности на практическом уровне. Можно ожидать, что модели активных Дх будут нормально работать на частотах до 1-10 МГц, а пассивных ДХ - на частотах до 100 МГц и более. Однако с повышением частоты увеличивается фазовая погрешность модели (см. рис. 7в), что несколько сужает область ее потенциального использования. Это происходит за счет частичной компенсации фазовых сдвигов в индукционном и емкостных звеньях модели.

Важнейшей составляющей разработки моделей компонентов является методология идентификации их параметров, причем экспериментальное определение многих из них требует привлечения достаточно сложного метрологического обеспечения, в Т. ч. средств измерений. Результаты измерений, выполняемых для обеспечения идентификации параметров моделей, во многом определяют последующую точность результатов моделирования. Для выполнения таких измерений целесообразно использовать современные средства измерений ведущих мировых фирм-производителей, например компании Rohde\&Schwarz, как это было сделано выше при идентификации динамических параметров активного датчика Холла.

\section{ЛИТЕРАТУРА}

1. Алексеев О. В., Головков А. А., Пивоваров И. Ю. и др. Автоматизация проектирования радиоэлектронных средств. Учебное пособие для вузов / Под ред. Алексеева О.В. - М.: Высшая школа, 2000. 479 с.

2. Левшина Е.С., Новицкий П. В. Электрические измерения физических величин. Измерительные преобразователи. Учебное пособие для вузов. - М.: Энергоатомиздат, 1983. 320 C

3. Лемешко Н. В., Кечиев Л. Н., Захарова С. С. IBIS-модели и их применение в задачах ЭМС. - М.: Грифон, 2016. 192 с.

4. Аполлонский С. М. Справочник по расчету электромагнитных экранов. - Ленинград: Энергоатомиздат, 1988. 224 с.

5. Кечиев Л. Н., Акбашев Б. Б., Степанов П. В. Экранирование технических средств и экранирующие системы. М.: ООО «Группа ИДТ», 2010. 470 с

6. Дао Динь Ха, Стемпицкий В.Р. Частотные характеристики интегральных датчиков Холла // Доклады БГУИР, сборник научных статей. 2018. № 4 (114). С. 64-70.

7. Лемешко н.В. Функционально-интерфейсные модели радиоэлектронных средств // Труды НИИР, сборник научных статей/Под ред. Бутенко В. В. - М.: НИИР. 2010, № 2. C. $65-72$.

8. Solid State Hall Effect Sensors. High Performance Miniature Ratiometric Linear. S\$490 Series. - Интернетpecypc www.honeywell.com/sensing (дата обращения 25.09.2019) 